\title{
MEGARA. High-precision alignment system for gluing fibers and microlenses
}

\author{
A. Pérez-Calpena ${ }^{a}$, E. Sánchez-Blanco ${ }^{\mathrm{a}}$, I. Martínez-Delgado ${ }^{\mathrm{a}}$, M.L. García-Vargas ${ }^{\mathrm{a}}$, X. Arrillaga ${ }^{\mathrm{b}}$, \\ H. Aube ${ }^{\mathrm{c}}$, A. Gil de Paz ${ }^{\mathrm{d}}$, M. Maldonado ${ }^{\mathrm{a}}$, J. Gallego ${ }^{\mathrm{c}}$, J. Iglesias-Páramo ${ }^{\mathrm{e}}$, E. Carrasco ${ }^{\mathrm{f}}$, F.M. \\ Sánchez-Moreno ${ }^{\mathrm{g}}$, J. Vilchez ${ }^{\mathrm{e}}$, R. Cedazo ${ }^{\mathrm{g}}$ \& MEGARA Team \\ ${ }^{a}$ FRACTAL SLNE, C/ Tulipán 2, Portal 13 1A, E-28231, Las Rozas de Madrid, Spain; \\ ${ }^{\mathrm{b}}$ AVS, Pol. Ind. Sigma Xixilion Kalea 2, Bajo Pabellón 10, E-20870, Elgoibar, Gipuzkoa, Spain; \\ ${ }^{\mathrm{c}}$ SEDI ATI Fibres Optiques 8 Rue Jean Mermoz, 91080 Courcouronnes, France; \\ ${ }^{d}$ GUAIX Group, Astrophysics Department, Universidad Complutense de Madrid, Av. Complutense \\ s/n, E-28040, Madrid, Spain; \\ ${ }^{\mathrm{e}}$ Instituto de Astrofísica de Andalucía, Glorieta de Astronomía s/n, E-18008, Granada, Spain; \\ fInstituto Nacional de Astrofísica, Óptica y Electrónica, Luis Enrique Erro \#1, Tonanzintla, Puebla, \\ 72840, Mexico; \\ ${ }^{\mathrm{g}}$ Facultad de Informática, Universidad Politécnica de Madrid, Campus de Montegancedo, E-28660, \\ Boadilla del Monte, Madrid, Spain;
}

\begin{abstract}
MEGARA is the multi-object medium-resolution spectrograph for the GTC 10m telescope. MEGARA offers two observing modes, the LCB mode, a large central IFU; and a MOS mode composed by 92 robotic positioners carrying 7 fibers minibundles. Microlens are required to fit the GTC f/17 to the $f / 3$ at the fiber entrance, where pupil image is oversized to have a fiber-to-fiber flux variation better than $10 \%$. This tight requirement imposed manufacturing tolerances for the different components and required the development of a gluing station to provide a centering precision better than $5 \mu \mathrm{m}$. We present the overview of the optical bundles, the gluing station and the final performance obtained during the integration and tests.
\end{abstract}

Keywords: MEGARA, integral field unit (IFU), multi-object spectrograoh (MOS), fiber bundles, fiber and microlens gluing station, high precision alignment, fibre-to-fibre flux homogeneity, GTC

\section{INTRODUCTION}

MEGARA (Multi Espectrógrafo en GTC de Alta Resolución para Astronomía) is an optical fiber fed spectrograph. The MEGARA focal plane subsystems are located at one of the Folded Cassegrain focal stations of the Spanish $10.4 \mathrm{~m}$ telescope GTC. Fiber bundles conduct the lights to the spectrograph that will be hosted at one of the Nasmyth platform (see Figure 1). The instrument offers two observing modes: the integral field units (IFUs), named as the Large Compact Bundle (LCB), and multi-object spectrograph (MOS). Both modes are fed by $100 \mu \mathrm{m}$ fiber core. The IFU provides a FOV of $12.5^{\prime \prime} \times 11.3^{\prime \prime}$, while the MOS shall be able to place up to 100 mini-bundles with 7 fibers each $(100 \mu \mathrm{m}$ fibercore) covering a sky area of $3.5 \times 3.5 \mathrm{arcmin}^{2}$. The science light will come into the spectrograph from one of the Folded Cassegrain focus (where the LCB and MOS subsystems are located and telecentrically illuminated with the aims of a field lens), by means of a $44.5 \mathrm{~m}$ fiber optic wiring. At the fiber entrance the telescope focal ratio is reduced from F17 to F3 with the aim of a micro-lens array. Subsequently each fiber bundle will be allocated at the spectrograph focal plane forming a smoothly curved pseudo-slit with a radius of curvature of $1075 \mathrm{~mm}$ and $110 \mathrm{~mm}$ long.

Advances in Optical and Mechanical Technologies for Telescopes and Instrumentation II, edited by Ramón Navarro, James H. Burge, Proc. of SPIE Vol. 9912, 99125L

(c) 2016 SPIE - CCC code: 0277-786X/16/\$18 - doi: 10.1117/12.2232620 

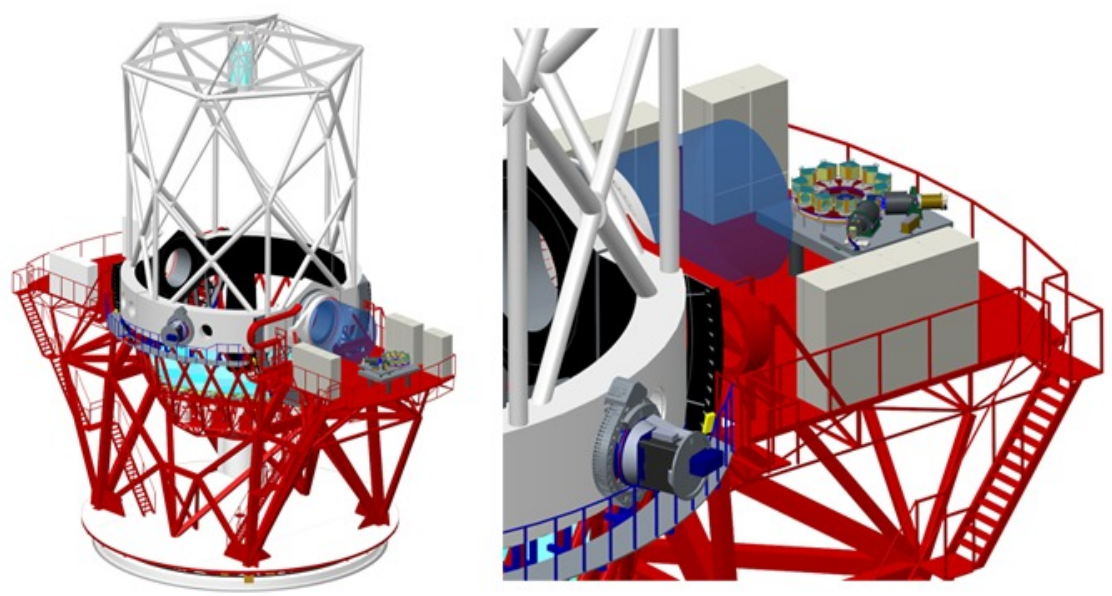

Figure 1: Left hand side shows a perspective of GTC with MEGARA placed at one of the Nasmyth platform while right hand side shows a zoom where the Folded Cassegrain focus and MEGARA on the Nasmyht platform are seen.

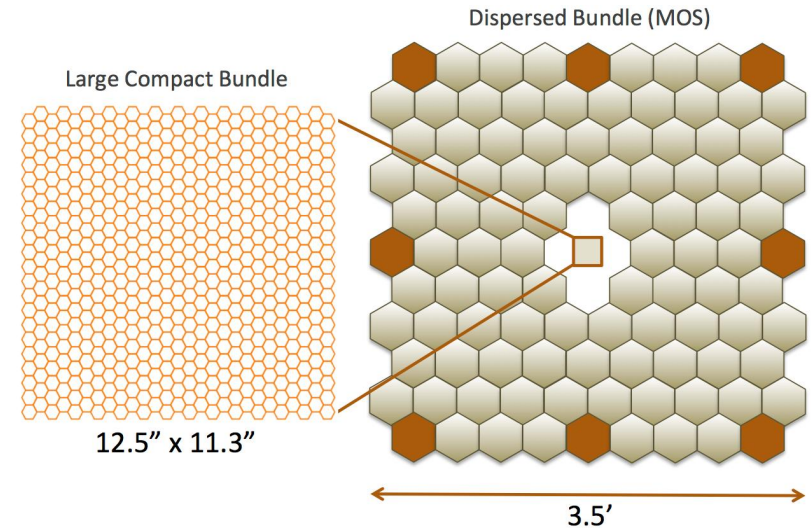

Figure 2: MEGARA FOV layout.

MEGARA shall work in the optical range from $3700 \AA$ to $9800 \AA$. The spectrograph is an all refractive system (with F3 and F1.5 focal ratios for the collimator and camera respectively) using a set of interchangeable gratings that are based on volume phase hologram gratings (VPHs) placed at the collimated beam (where the pupil of the system is formed). The final set offer three spectroscopy modes: at low, medium and high resolution (accordingly to $\mathrm{R}_{\mathrm{FWHM}}=6000,12000$, 18700). Finally, the scientific data will be recorded by means of an E2V $4 \mathrm{k} \times 4 \mathrm{k}$ detector, with a $15 \mu \mathrm{m}$ pixel size. The main parameters are shown in Table 1.

The Universidad Complutense de Madrid (UCM) leads the MEGARA Consortium. The rest of MEGARA Consortium partners is constituted by the Instituto Nacional de Astrofísica, Óptica y Electrónica (INAOE) in Mexico, and the Instituto de Astrofísica de Andalucía (IAA-CSIC) and the Universidad Politécnica de Madrid (UPM) in Spain.

Table 1: Main characteristics of MEGARA LCB IFU and MOS modes

\begin{tabular}{|l|c|}
\hline \multicolumn{1}{|c|}{ PARAMETER } & REQUIREMENTS \\
\hline Telescope & GTC \\
\hline Plate Scale & $0.82 \mathrm{~mm} \mathrm{arcsec}^{-1}$ \\
\hline Field Lens & $\mathrm{D}=260 \mathrm{~mm}$ \\
\hline Microlens & $\begin{array}{c}\text { Hexagonal Shape } \\
\text { F-ratio transformation from F17 to F3 }\end{array}$ \\
\hline
\end{tabular}




\begin{tabular}{|c|c|c|c|}
\hline \multirow{2}{*}{\multicolumn{2}{|c|}{ Fiber Unit }} & \multicolumn{2}{|c|}{$\begin{array}{l}\text { Holded at Folded Cassegrain focus } \\
\text { Field lens for telecentric illumination } \\
\text { LCB, SCB, MOS }\end{array}$} \\
\hline & & Large Compact Bundle & MOS Bundle \\
\hline \multicolumn{2}{|c|}{ Fiber-core } & $100 \mu \mathrm{m}$ & $100 \mu \mathrm{m}$ \\
\hline \multicolumn{2}{|c|}{ No. of fibers } & 623 & 644 \\
\hline \multicolumn{2}{|c|}{ Spaxel } & $0.62^{\prime \prime}$ & $0.62^{\prime \prime}$ \\
\hline \multicolumn{2}{|c|}{ FOV } & $12.5^{\prime \prime} \times 11.3^{\prime \prime}$ & $3.5^{\prime} \times 3.5^{\prime}$ \\
\hline \multicolumn{2}{|c|}{$\Delta \lambda\left(\mathbf{E E D}_{\mathbf{8 0}}\right)$} & 4.0 pix & 4.0 pix \\
\hline \multicolumn{2}{|c|}{$\Delta \lambda($ FWHM) } & 3.6 pix & 3.6 pix \\
\hline \multirow{3}{*}{$\mathbf{R}$} & LR & 6000 & 6000 \\
\hline & MR & 12000 & 12000 \\
\hline & HR & 18700 & 18700 \\
\hline \multicolumn{2}{|c|}{ Pseudo-slit } & \multicolumn{2}{|c|}{$110 \mathrm{~mm}$ long \& $\mathrm{ROC}=1075 \mathrm{~mm}$} \\
\hline \multicolumn{2}{|c|}{ F-ratios } & \multicolumn{2}{|c|}{ Collimator F3 to camera F1.5 } \\
\hline \multicolumn{2}{|c|}{ Pupils size } & \multicolumn{2}{|c|}{$160 \mathrm{~mm}$} \\
\hline \multicolumn{2}{|c|}{ Gratings } & \multicolumn{2}{|c|}{ VPHs, $240 \mathrm{~mm} \times 190 \mathrm{~mm}(\mathrm{MR}, \mathrm{HR}), 220 \mathrm{~mm} \times 180 \mathrm{~mm}(\mathrm{LR})$, selectable } \\
\hline \multicolumn{2}{|c|}{ Wavelength coverage } & \multicolumn{2}{|c|}{$3700 \AA-9800 \AA$} \\
\hline \multicolumn{2}{|c|}{ Detector } & \multicolumn{2}{|c|}{ E2V $1 \times 4 \mathrm{k} \times 4 \mathrm{k}, 15 \mu \mathrm{m}$ pixel size, AR coated } \\
\hline
\end{tabular}

The MEGARA Science Team includes a line-up expert researchers encompassing different astronomical areas from exoplanets \& cool stars to cosmology, going through massive stars, planetary nebula, and nearby galaxies, coming from all the MEGARA consortium institutions and other Spanish and Mexican research centers together the University of Florida (FL).

MEGARA is being developed under contract with GRANTECAN. MEGARA is finalizing the integration and verification phase and the delivery to GTC is scheduled in December 2016.

Section 2 briefly describes the MEGARA focal plane subsystems. Section 3 summarizes the flux homogeneity error budget that was prepared to analyze and control the optical performance of the fiber-microlens system, while section 4 presents the gluing system and process developed for the MEGARA project to provide the alignment precision required between fibers and microlens and the results obtained during the integration and verification phase.
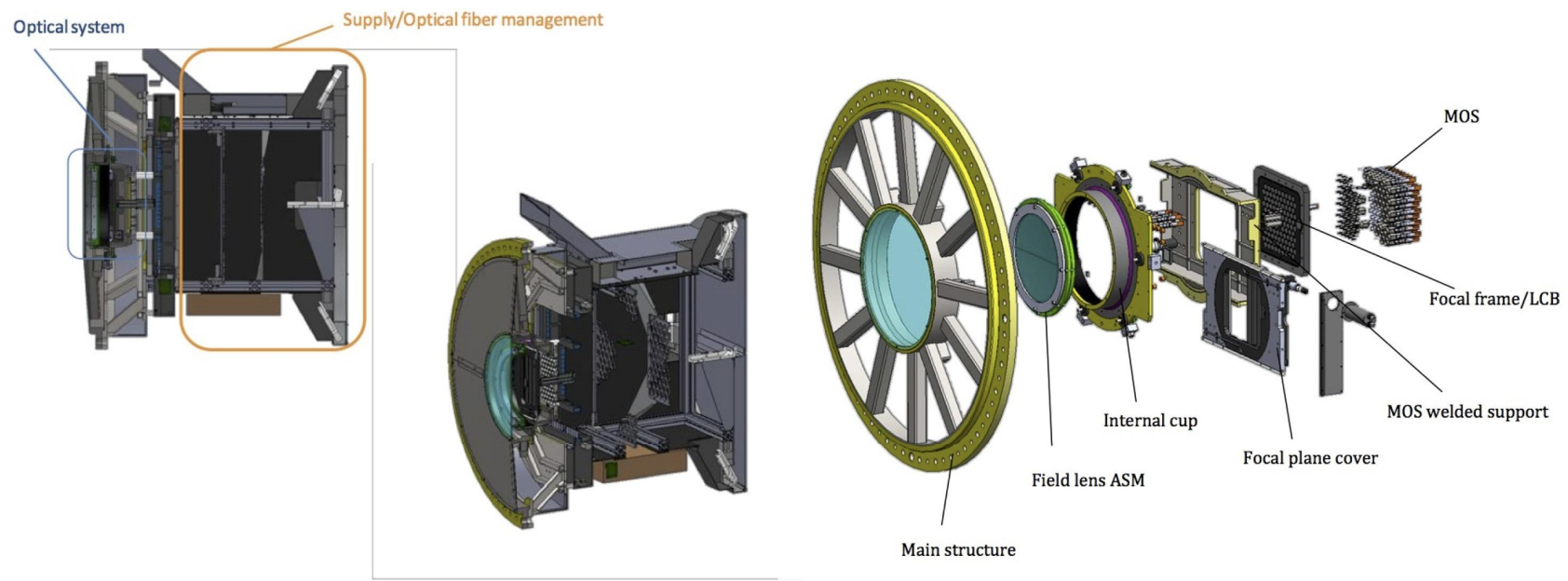

Figure 3: Section of the Folded Cassegrain subsystems assembly and a exploded view of the optical components. 


\section{FOCAL PLANE SUBSYSTEMS}

All these subsystems are found at the Folded Cassegrain rotator adapter, a structure that fixes and positions the different optical subsystems of the focal plane at the Folded Cassegrain focus station including the field lens, the focal plane cover, the positioners and the IFU. Figure 3 shows a section of the Folded Cassegrain subsystems assembly and an exploded view of the optical components. The optical and electrical cable management subsystems, which are in charge of routing optical and electrical cables to the Folded Cassegrain rotator, are also included within the adapter.

The MEGARA Folded Cassegrain adapter support the following subsystems:

- The field lens shall correct the lack of telecentricity of the GTC focal station providing a field curvature below 0.1 in the whole FOV.

- The focal plane cover shall allow occulting part of the fibers (LCB and MOS) for performing null-cross-talk observations. A Focal-Plane Cover would be used so to allow removing the light coming from one every two consecutive fibers (or sets of fibers) at the corresponding pseudo-slit. In the case of the LCB this implies arranging the fibers so two consecutive fibers will come from different halves of the FOV. Regarding the MOS the decision was that all seven fibers from each MOS would be placed together in the pseudo-slit (to minimize inter-positioner cross-talk between the central - brightest - fiber of each positioner) but that adjacent sets of 7fiber would come, again, from different halves of the FOV.

- The 2D refractive micro-lens arrays shall couple the science light at the telescope focal plane into the fibers, defining the FOV and adapting F\# from F17 to F3 to minimize FRD. The micro-lens arrays are arranged in different arrays for each mode following a hexagonal geometry to maximize the area to be cover. The spaxel size shall be fixed to $0.62^{\prime \prime}$.

- The LCB is an IFU bundle compound of 567 fibers (100 $\mu \mathrm{m}$ fiber-core, spaxel $\left.0.62^{\prime \prime}\right)$ delivering a FOV of 12.5" $\times 11.3^{\prime \prime}$ near the optical axis of the instrument plus 8 positioner robots located in the outer edge of the instrument FOV that let simultaneously sky background measurements (see Figure 2 and Figure 5).

- The MOS bundle is composed by the fibers coming from 92 robotic positioners (see Figure 2) that allows to position the 7 fibers minibundles carried by each positioner anywhere in the $3.5 \times 3.5 \operatorname{arcmin}^{2}$ FOV. Both LCB and MOS bundles have been integrated by SEDI (see Figure 5).

- The Fiber MOS shall consist of 100 robotic positioners (8 of them for LCB sky subtraction). Each fiber minibundle patrols a circular area of diameter $\Phi 23.21 \mathrm{~mm}$ thanks to the two rotations provided by the positioner robot (see Figure 4). The positioner was designed and manufactured by AVS.

- The fibers bundles are arranged in two rows at the pseudo-slit located at the spectrograph entrance. Each pseudoslit is divided in boxes to simulate the focal plane curvature at the spectrograph entrance (ROC $1075 \mathrm{~mm}$, size $110 \mathrm{~mm}$ ).

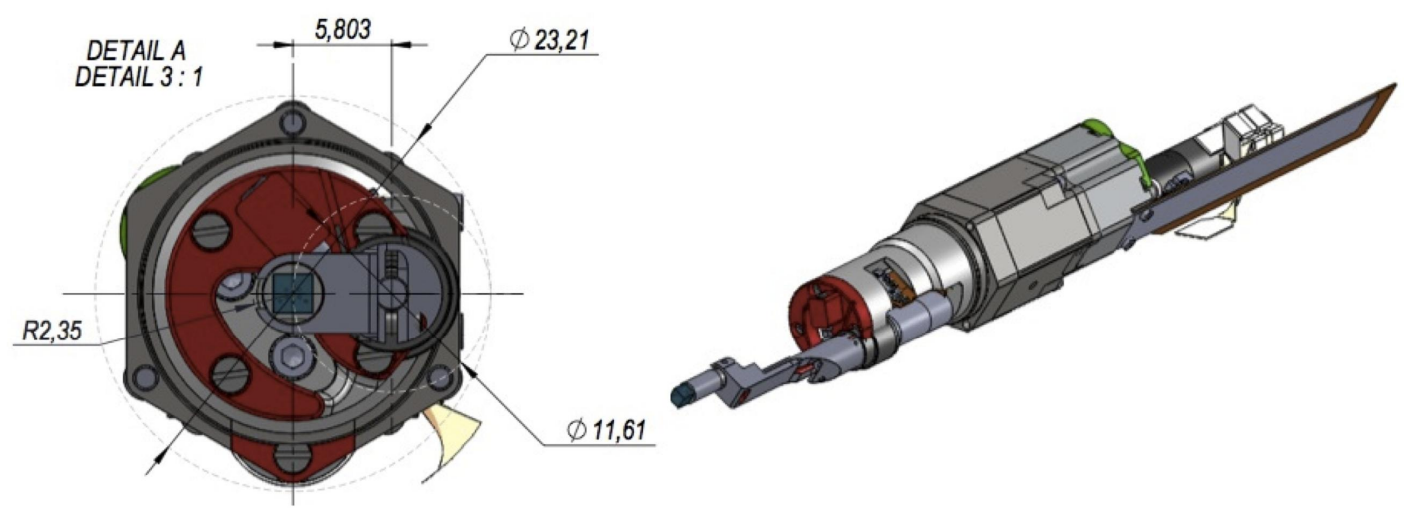

Figure 4: Image of the robotic positioner of MEGARA MOS (AVS). 


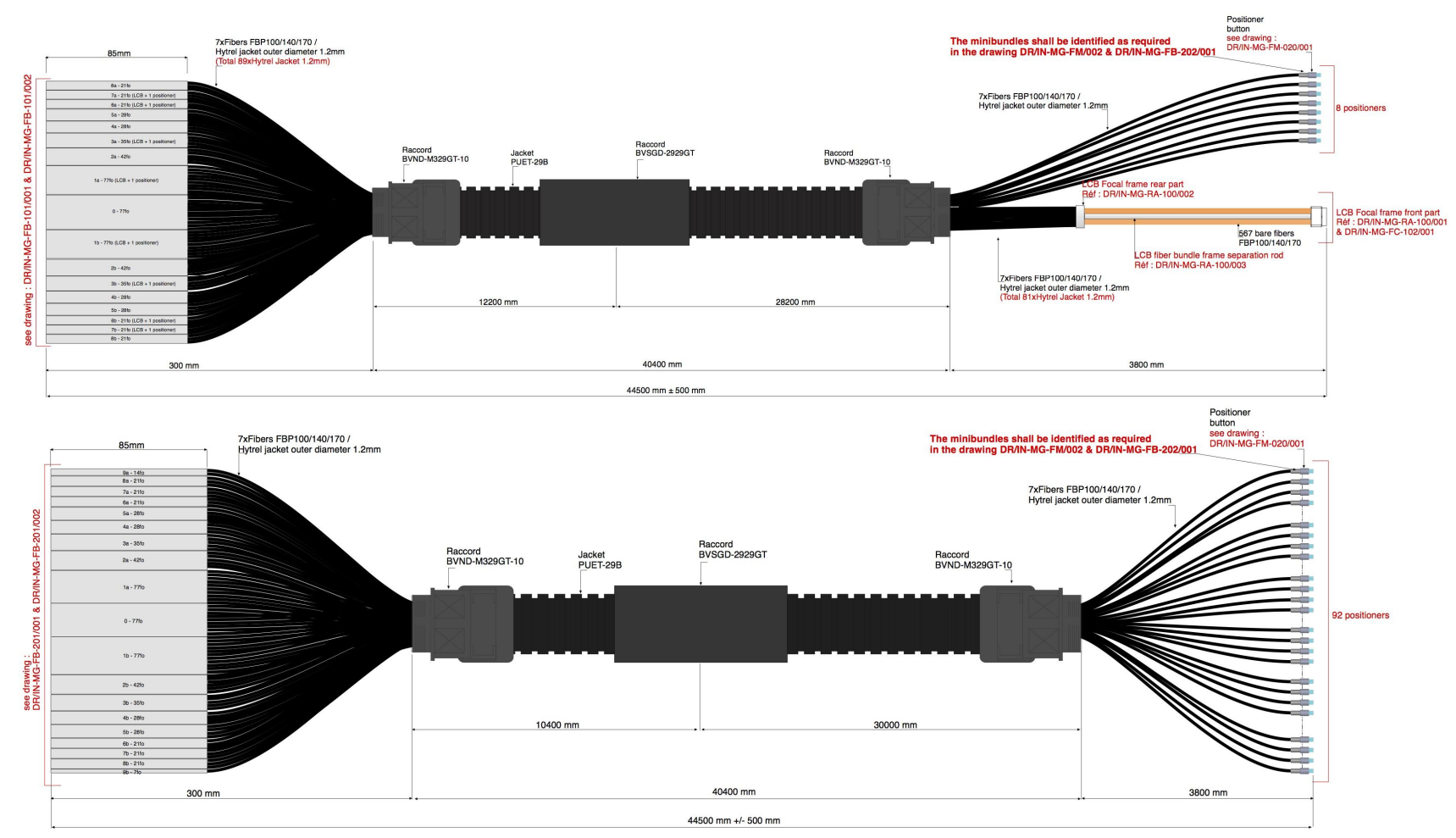

Figure 5: LCB (up) and MOS (down) fiber bundles drawings (SEDI).

\section{FLUX HOMOGENEITY ERROR BUDGET}

As mentioned in the previous section, the microlens arrays shall define the FOV to be introduced in the fibers, adapting F\# from f/17 (GTC telescope) to $\mathrm{f} / 3$ (Fibers) to minimize FRD and to provide a telescope pupil image on the $100 \mu \mathrm{m}$ fiber core. The spaxel size is 0.62 arcsec oversizing the pupil image and finding a compromise to optimize the following parameters:

a) the flux recovered from point sources with each MEGARA MOS mini-bundle,

b) the fraction of lost light when reimaging the pupil on the fiber core,

c) the need of fully imaging the fiber core in order to preserve the quality of the fiber-to-fiber relative-flux calibration and

d) the differential atmospheric refraction effects that has to be minimized.

If the full fiber core was not illuminated, differences between spectral calibration response and the science object regarding the spectral lines centroids could be found and, besides, any misalignment would introduce different transmission response among the fibers. Overfilling the pupil image allows providing an illumination condition as similar as possible to the one given by the spectral calibration system (that is particularly important should a $\mathrm{f} / 17$ beam not be available at the Instrument Calibration Module) and to minimize mounting or assembly errors between fibers due to misalignment or other.

The requirement on the flux-calibration stability of the system due to potential fiber-to-fiber misalignments among the pupil images on each fiber core is a $<10 \%$ fiber-to-fiber variation in the fraction of the pupil imaged so to ensure a proper relative-flux calibration and cosmetics. This requirement drives, on the one hand, the selection of the spaxel size and, on the other hand, the tolerances to the mounting and assembly errors, which impose a $10 \mu \mathrm{m}$ tolerance for positioning the pupil image on the fiber core.

A detailed error budget, called Flux Homogeneity error budget, was prepared for deriving the manufacturing tolerances 
of the frames supporting the fibers, the microlens diameters and positions at the array, the external fiber diameters, the positioning accuracy of the robotic positioner and, the performance of the gluing system between fiber and microlens.

\subsection{Flux homogeneity error budget at the detailed design phase}

The different contributors to build this error budget were estimated at the detailed design as shown at Table 2 .

Table 2: Flux homogeneity error budget (Detailed design).

\begin{tabular}{|l|c|c|}
\hline ITEM & LCB mode & MOS mode \\
\hline Microholes position & $\pm 5 \mu \mathrm{m}$ & $\pm 5 \mu \mathrm{m}$ \\
\hline Microholes diameter (fiber centering) & $\pm 3.5 \mu \mathrm{m}$ & $\pm 3.5 \mu \mathrm{m}$ \\
\hline Microlens position & $\pm 5 \mu \mathrm{m}$ & $\pm 5 \mu \mathrm{m}$ \\
\hline Microlens diameter & $\pm 2 \mu \mathrm{m}$ & $\pm 2 \mu \mathrm{m}$ \\
\hline Microlens - fiber assembling and gluing & $\pm 5 \mu \mathrm{m}$ & $\pm 5 \mu \mathrm{m}$ \\
\hline Support frame assembling errors (static) & $\pm 1 \mu \mathrm{m}$ & $\pm 1 \mu \mathrm{m}$ \\
\hline MOS minibundles dynamic errors & - & $\pm 4.7 \mu \mathrm{m}$ \\
\hline TOTAL & $\pm \mathbf{9 . 5 5} \mu \mathrm{m}$ & $\pm \mathbf{1 0 . 6 9} \mu \mathrm{m}$ \\
\hline Requirement & $\pm \mathbf{1 0} \boldsymbol{\mu m}$ & $\pm \mathbf{1 0} \mu \mathrm{m}$ \\
\hline
\end{tabular}

- Microholes position and diameter: The LCB frame (see Figure 6) and the positioner buttons were manufactured by AVS. The dimensional verification reports of those parts confirmed that the microholes were positioned with a tolerance better than $\pm 5 \mu \mathrm{m}$ and that fiber could be centered with a tolerance better than $\pm 3.5 \mu \mathrm{m}$.

- Microlens position and diameter: The microlens arrays were manufactured by AMUS. The verification report provided by AMUS confirmed that the microlens were positioned with a tolerance better than $\pm 5 \mu \mathrm{m}$ and the diameter was as specified with a tolerance better than $\pm 2 \mu \mathrm{m}$.

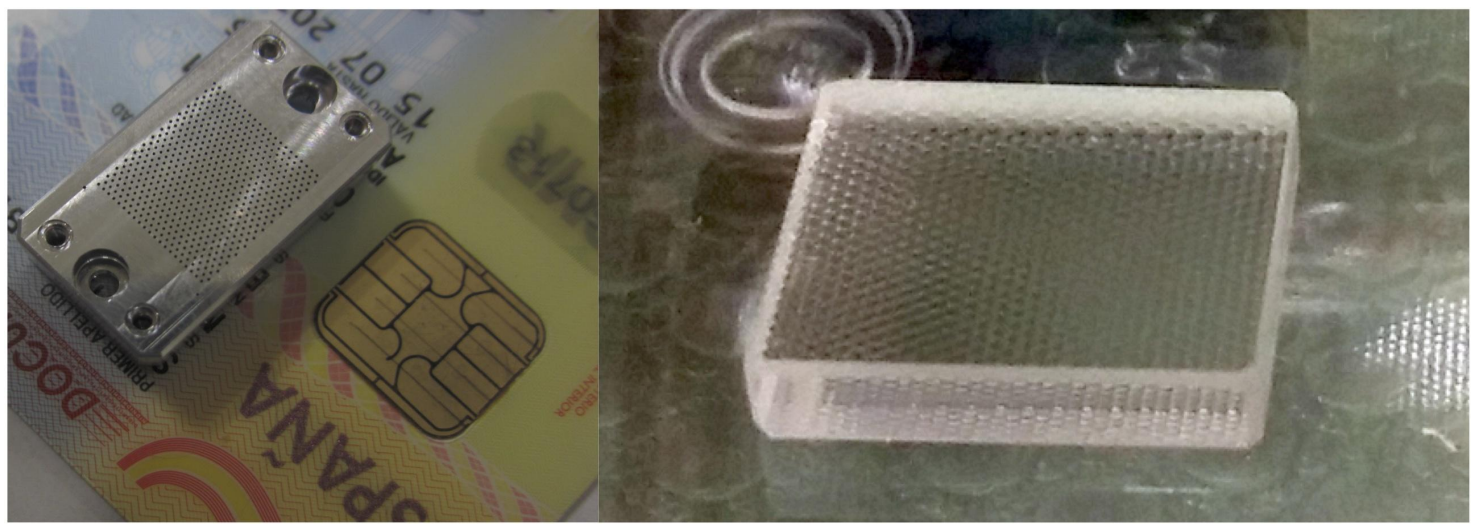

Figure 6: Left. LCB frame (AVS). Right. LCB microlens array (AMUS). 
- Microlens-fiber assembly and gluing: The gluing station was designed to achieve a tolerance in the microlensfiber alignment process better than $\pm 5 \mu \mathrm{m}$. The gluing station and process that was followed is described in detail in Section 4.

- Support frame assembly errors (static): Once the microlens arrays are glued at the LCB frame and MOS buttons, the buttons are glued at the positioners and, then, all positioners and the LCB frame are assembled at the Fiber MOS focal plane, which is finally integrated at the rotator adapter frame. In both cases (LCB and MOS), it was estimated that the assembly error would be better than $\pm 1 \mu \mathrm{m}$.

- MOS minibundle dynamic error: The error of the MOS minibundles due to the positioner rotations was estimated to be lower than $\pm 4.7 \mu \mathrm{m}$ (based on the measurements performed at the positioner prototype developed at the preliminary design).

\subsection{MOS flux homogeneity error budget at the AIV phase}

The contributors to the MOS flux homogeneity error budget were reviewed later at the AIV phase taking into account the following issues:

- An independent system was used to measure the tilt error after gluing fibers and microlens. The position error is then computed from the tilt error taken into account the microlens effective focal length. This independent system is measuring the total assembly error between fibers and microlens, which includes not only the error introduced by the gluing process but also the previous contributors coming from microlens and frames (LCB and buttons) manufacturing and fibers alignment at the microholes.

- The support frame assembly errors for the MOS system were reviewed considering the button-positioners gluing tests done at AVS.

- The dynamic limit error is maintained. Otherwise, changes have been done to improve positioners that lead to have better dynamic tilt results for the first positioner manufactured than initially obtained for the positioner prototype done at preliminary design.

Table 3: Flux homogeneity error budget (AIV).

\begin{tabular}{|l|c|}
\hline ITEM & MOS mode \\
\hline Microlens - fiber assembling and gluing & $\pm 7.3 \mu \mathrm{m}$ \\
\hline Support frame assembling errors (static) & $\pm 4.9 \mu \mathrm{m}$ \\
\hline MOS minibundles dynamic errors & $\pm 4.7 \mu \mathrm{m}$ \\
\hline TOTAL & $\pm \mathbf{9 . 9} \boldsymbol{\mu m}$ \\
\hline Requirement & $\pm \mathbf{1 0} \boldsymbol{\mu m}$ \\
\hline
\end{tabular}

Therefore, the requirement that must be fulfilled by the microlens - fiber assembly position error is to be less than \pm 7.3 $\mu \mathrm{m}$, which corresponds to \pm 14 arcmin tilt.

\section{GLUING SYSTEM}

\subsection{Gluing station}

This gluing system was designed with the aim to be able to manipulate the arrays during their positioning and gluing process onto the fiber button, and it is composed of a holder base able to grasp the fiber to be glued and allowing to 
move the fiber button with the following $R x, R y, R z$ and $x, y, z$ degrees of freedom. The gluing station includes an illumination system that will not only illuminate the array surface but will also provide a return image (with the aid of a beamsplitter) of the array onto a detector that will monitor and let us to control all the gluing process. More details about the design of this system is provided at [1].

When we performed the first tests for gluing the minibundle prototype, it was identified that some improvement from its initial design were required. These improvements have a direct impact in the control over the corrections at the X and Yaxes, XY tilt and RxRy. In this way, the most important changes that were implemented are:

- The micro-lens array is trapped on the flat reference for being glued (see Figure 7) avoiding the action of a torque that could introduce a tilt in the array + button system.

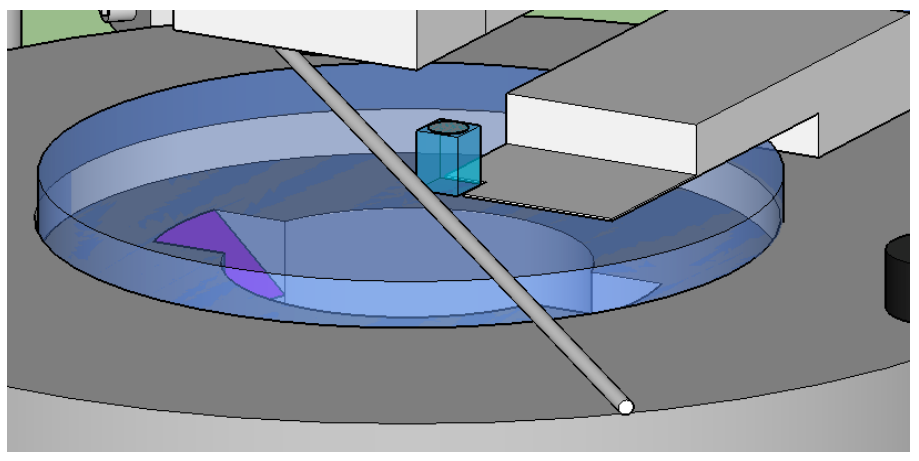

Figure 7: Microlens array trapped by the rod and the razor base

- The button is hosted in the corner formed between a flat mirror and a right angle prism (see Figure 8, right frame). This is because the mechanical surface reference is the cylindrical face of the button.

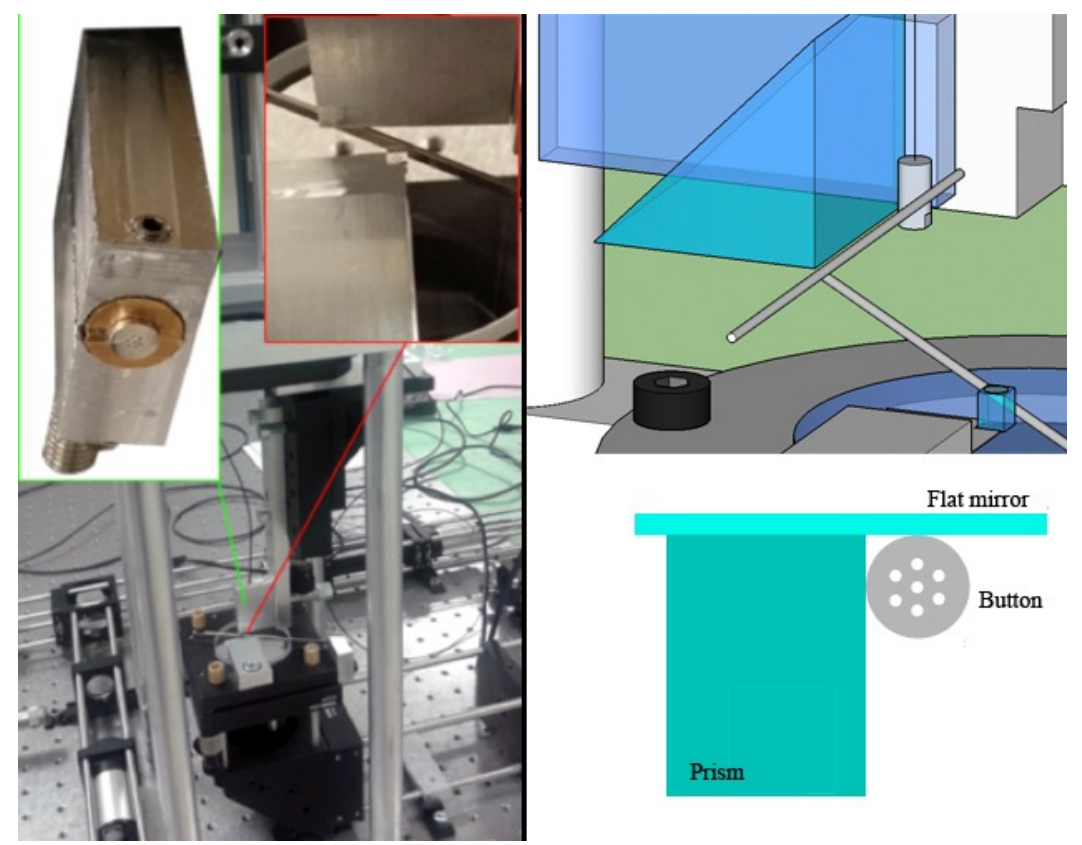

Figure 8: Left. Old version of the vertical arm of the gluing station from where the button and the optical fiber is going down towards the plate where the microlens array is fixed. Right top, button trapped by the system prism + flat mirror and a rod. Right bottom, the system seen from array. 
- The gluing station is provided with a custom base (as shown at Figure 7, where the array is hosted, that allows both $\mathrm{XY}$ tilt and $\mathrm{Rz}$ corrections (being $\mathrm{Z}$ axis that one defined by the optical fiber). Besides the vertical arm is allocated over a rotary stage allowing a $360^{\circ}$ rotation.

- A new detector was included to have an image of the final exit pupils from the system array + button. At this detector, we can see directly the effect of the fine rotation tunes and/or XY translation applied when the button and the array are in contact.

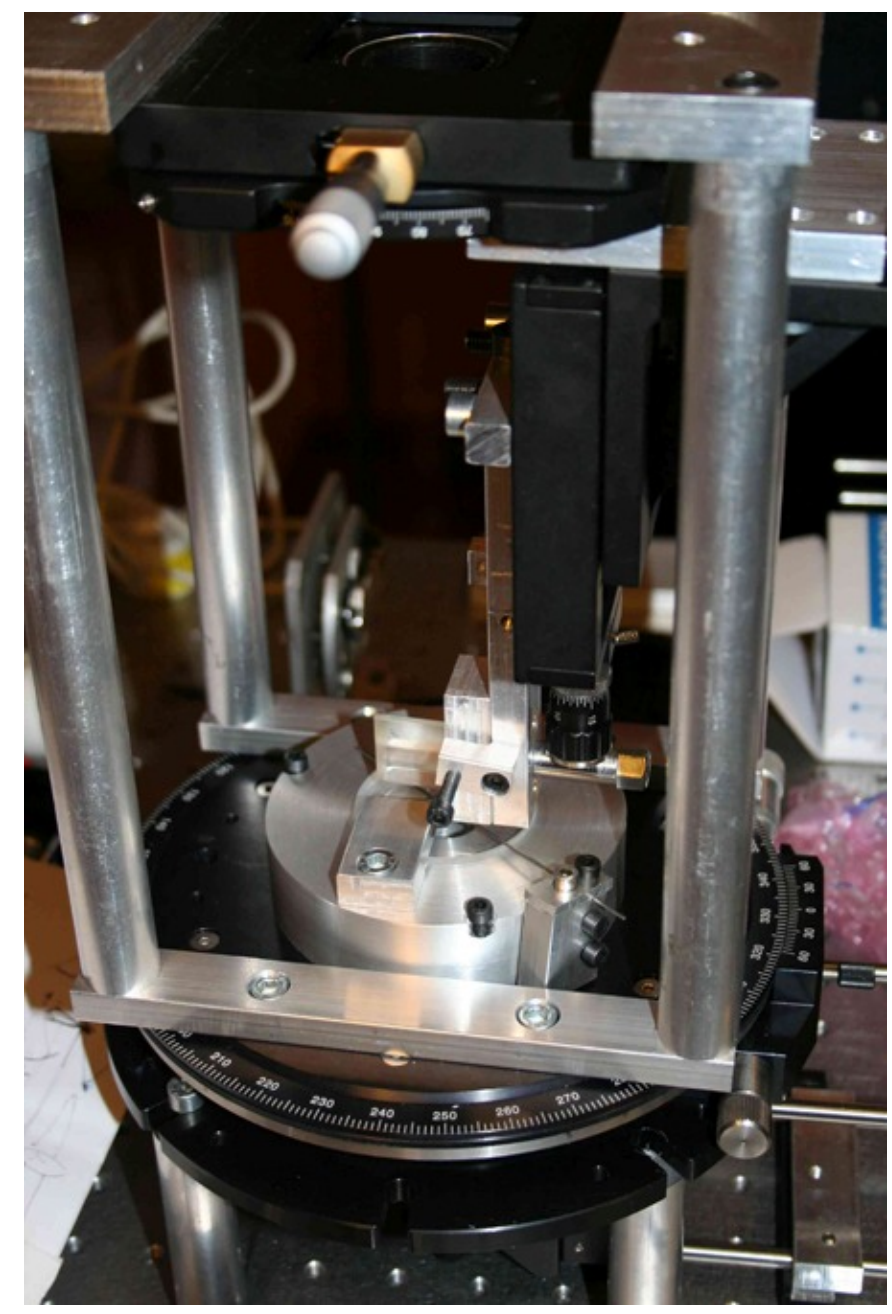

Figure 9: General view of the vertical arm of the GS, where the new rotary stage and the new custom base that supports the array are seen

\subsection{Gluing process}

The gluing process is accomplished as follows:

- The gluing stations alignment is performed using a laser beam. The aim is to ensure that the optical axis of the system is well defined, as well as the rotation axis of the gluing station vertical arm (Rz) and the fiber + button support (see Figure 10). 


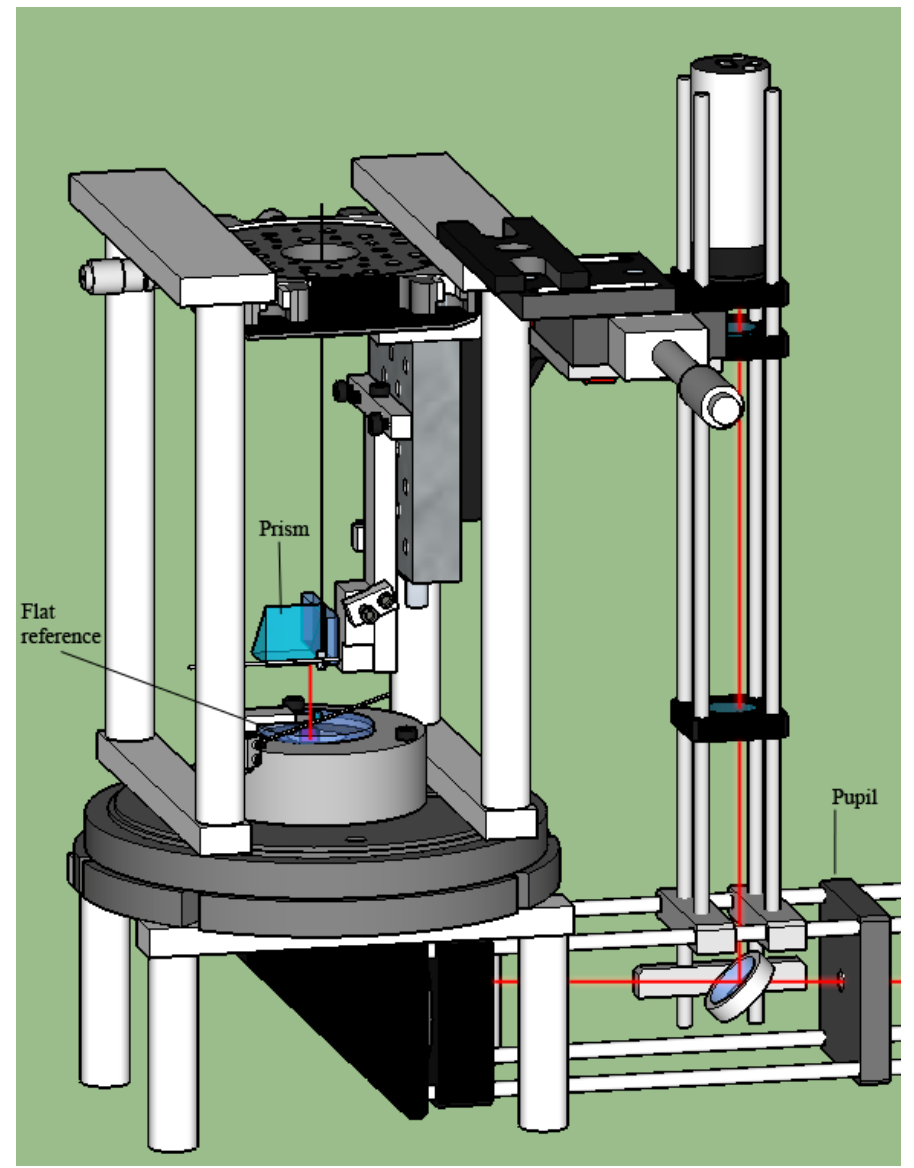

Figure 10: Alignment process of the button + fiber subsystem

- The pseudoslit is placed over the slit support and then is illuminated by the F17/F3 Illuminator unit. The pseudoslit is horizontally translated until the light is seen coming towards out the seven optical fibers of the button .

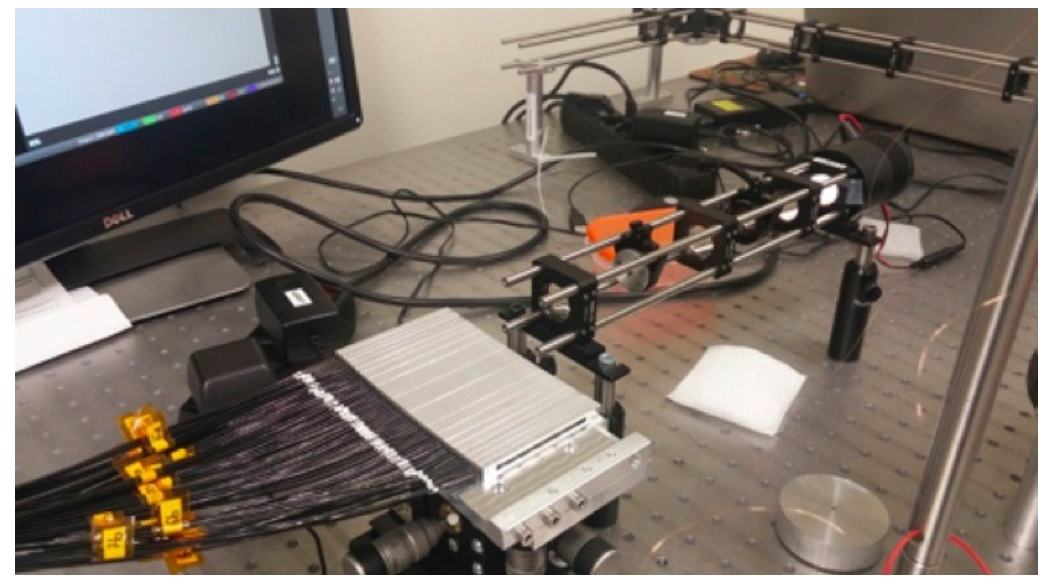

Figure 11: Pseudoslit illuminated by the F3/F17 IS unit and the exit at the button surface 
- The button is put against the walls defined by the flat mirror and the prism.

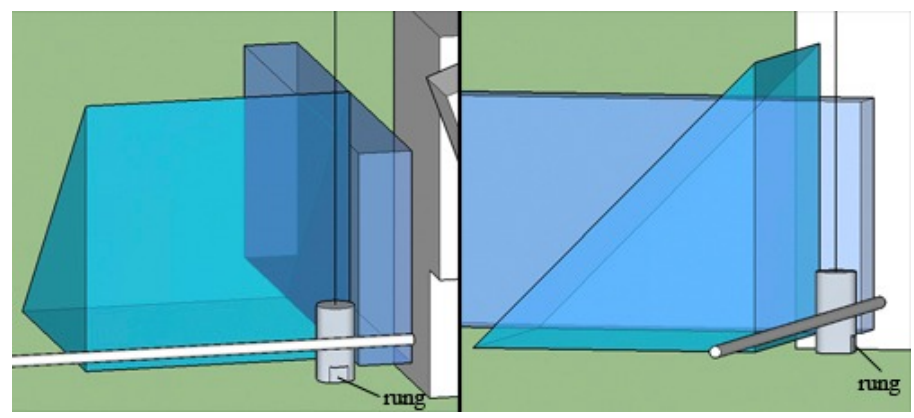

Figure 12: How to place the button during the gluing process

- The array is put with the microlenses making contact with the flat reference.

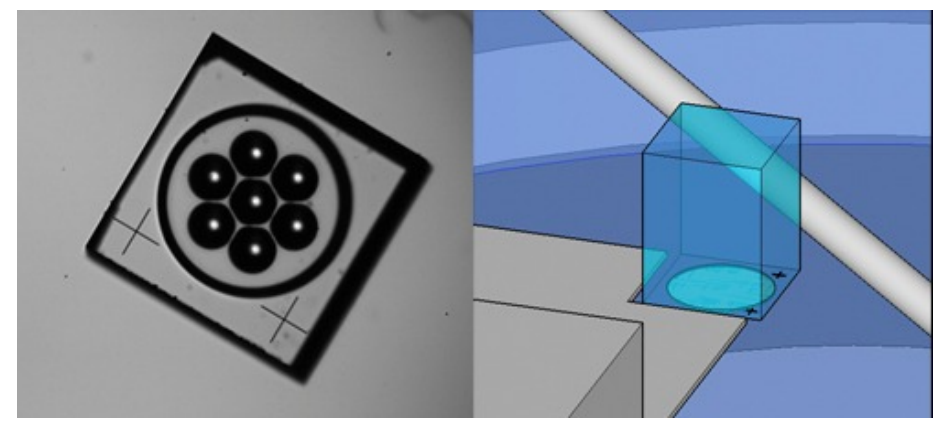

Figure 13: How to place the array during the gluing process

- Once the button and the array are put in their respective positions we follow with the button approximation to the FOV of the array as shown in Figure 14, until we overlap the light exiting from the fibers (pupils) over the microlenses.
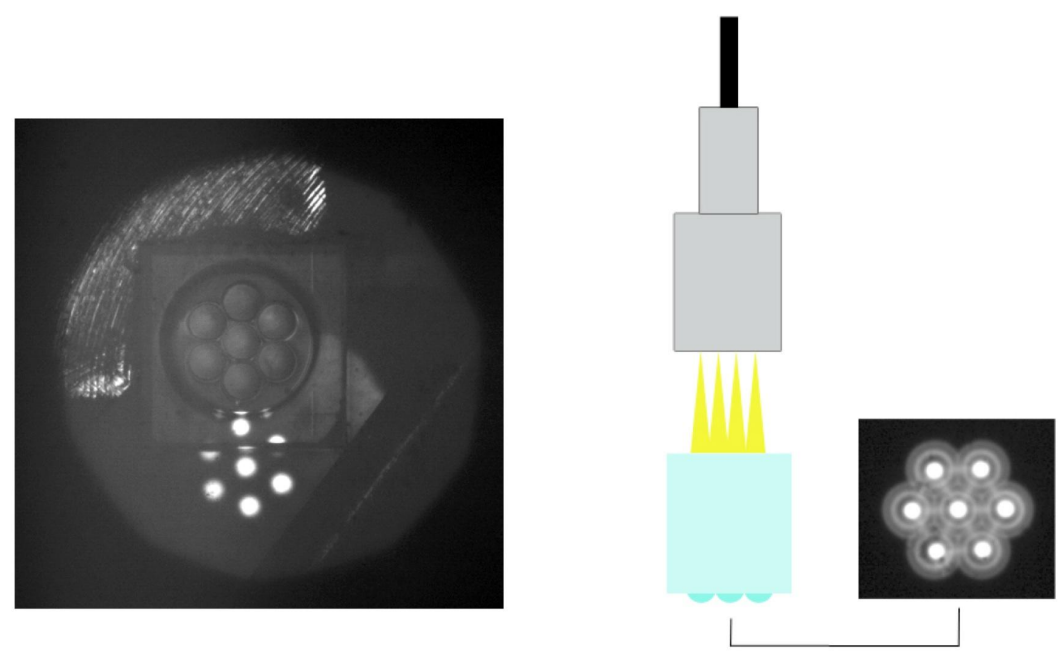

Figure 14: Button approximation to the optical axis of the microlens array (left). Image pattern seen when the button is going down facing the array (right). 
- This pattern will be very useful to make a first rotation approximation correction over the button position respect to the array. As we see from Figure 15 (left frame), the exiting pupils are not well centred to the optical axis of each microlens, therefore from this perspective is easier to apply the necessary rotational correction (Rz). Once applied this correction, the patter is centred as shown in Figure 15 (right frame).

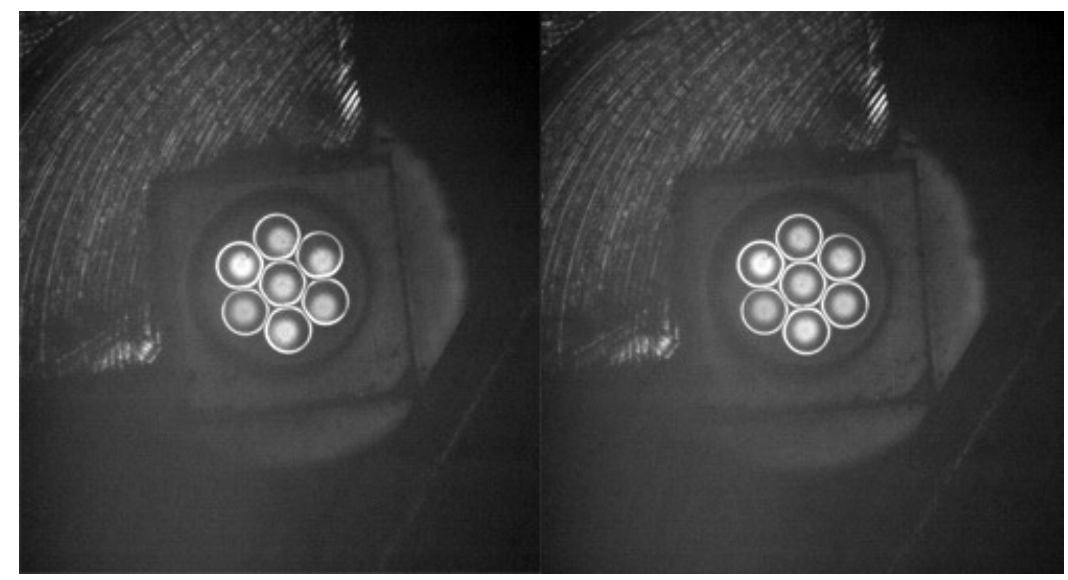

Figure 15: Rotation fine-tuning

- Then, the button is moved in one axis towards out the array view, to leave free space for applying the epoxy.

- The epoxy used during the gluing process is from Nordland, specifically the NOA85 with a refraction index that matches that of the Fused Silica, therefore very appropriate for avoiding Fresnel's looses between the optical fibers and the lenses. Due to the small dimensions we are treating on, it was selected a variable volume micropipette working in the range $0.1-2 \mu \mathrm{L}$. This will let the adhesive drop to be deposited with very high accuracy and precision. The final volume estimated $\sim 1.5 \mu \mathrm{L}$ to be optimal.

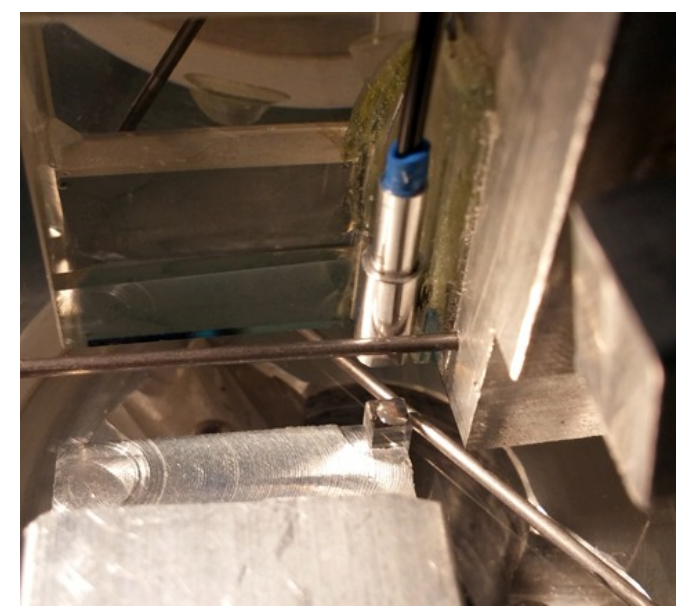

Figure 16: Microlens array with a 1.5 microliters drop on it

- After applying the epoxy we make the approximation to the array's surface. When the button makes contact with the array we see the pupils well uniformly illuminated and it is appreciated how the drop spreads over the surface. We continuous going down until the drop overflows the array's surface. 
- Once the contact has been made, we check the status of the seven pupils. Although previously we had applied a first rotation correction, now it could be necessary to carry out a new finer one at the level of the exiting pupils. If the pupils are not rightly overlapped as shown at Figure 17 (left), we should apply a fine rotation tune after the pupils are aligned and well overlapped, as shown in in Figure 17 (right). This rotation tune is performed together with a XY translation correction until the pupils do not move and stay statics at different gluing station vertical arm rotation changes
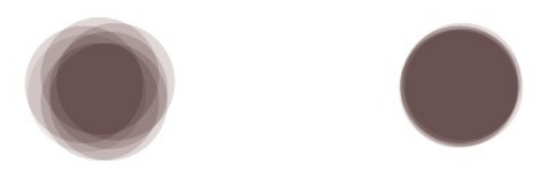

Figure 17: Image of the 7 pupils when the button has made contact with the array.

- Finally after all corrections are applied we cure the epoxy exposing it to a UV radiation coming from two LEDs installed bellow the flat reference where the array is laid on. The array + button is exposed during 5 minutes.
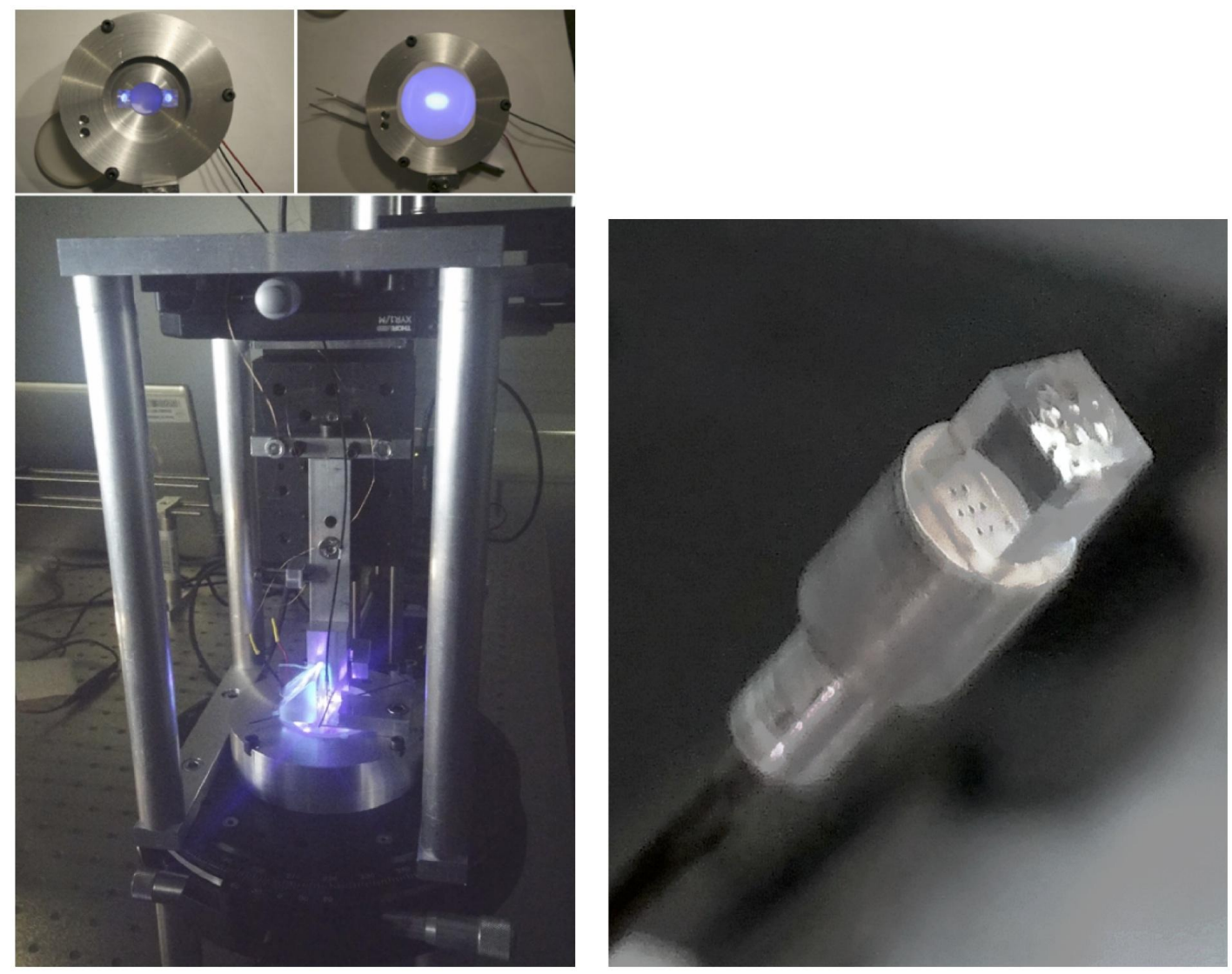

Figure 18: UV LEDs hosted at the base where the array is laid on (top left). UV radiation illuminated uniformly the flat reference where the array is hosted (top right). A glued array + button during the curing exposure. 
Similar steps are followed for gluing the LCB, which is finally cured as shown at Figure 20.
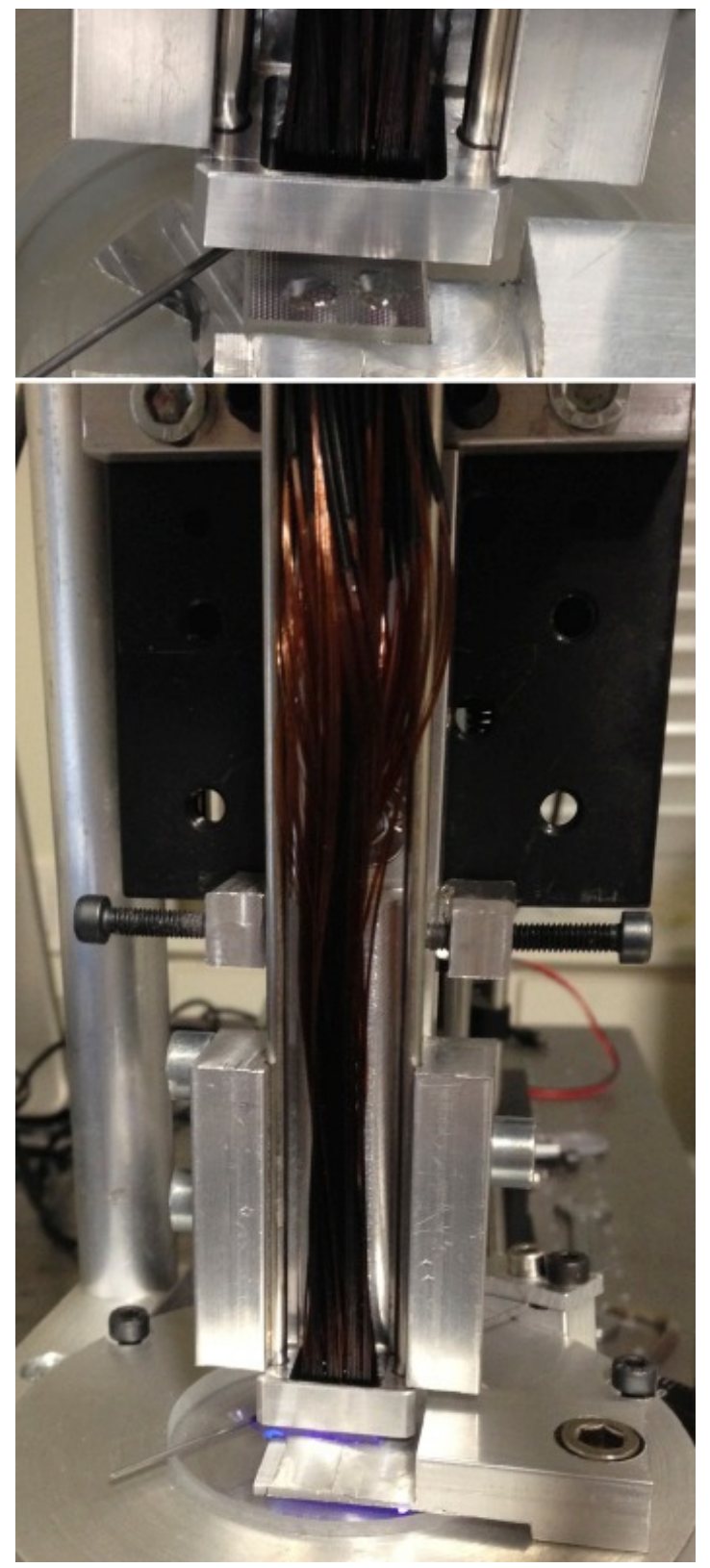

Figure 19: Epoxy drops on top of the microlens array (top). UV exposure during the epoxy curing.

\subsection{Gluing results}

As mention at section 3.2, an independent setup (see Figure 19) was prepared for measuring tilt errors. The button is put at the two flat mirrors in $\mathrm{V}$ position and is pressed over them by means of a steel rod to allow a complete surface's button contact over the mirrors, avoiding any artificial tilt. A $50 \mathrm{~mm}$ focal lens and a detector with a pixel size of $3.65 \mu \mathrm{m}$ compound the rest of the system. 


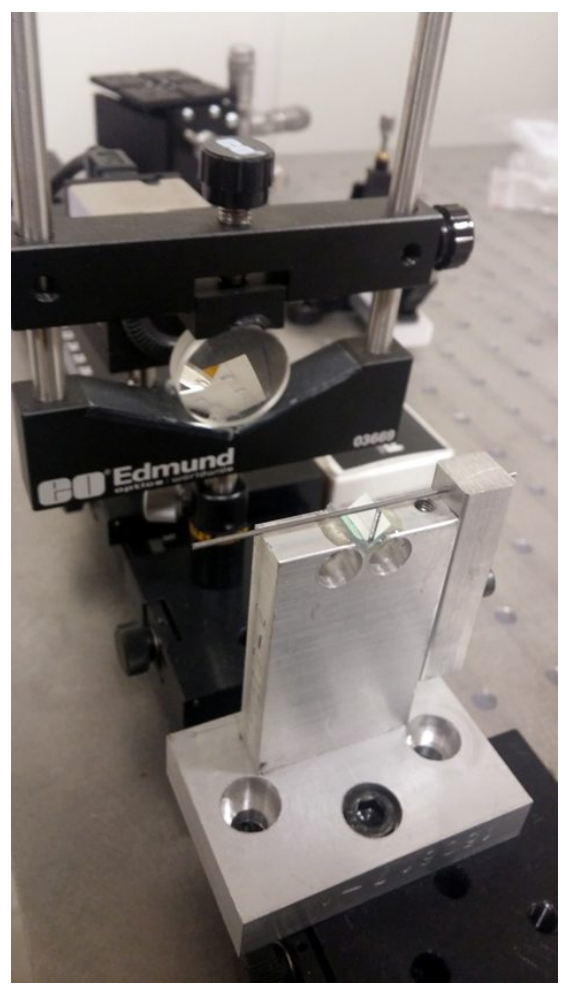

Figure 20: Tilt measurement setup.

All 100 minibundles + microlens measurements were below the 14 arcmin (see section 3.2). The tilt average measurement is $9.4 \operatorname{arcmin}(4.9 \mu \mathrm{m})$.

\section{SUMMARY}

The gluing of the MEGARA fiber bundles and microlens requires a high-precision system in order to meet the homogeneity flux requirement established for the system. The gluing station and process described in this article explains how it was accomplished to finally fulfill successfully this requirement.

\section{REFERENCES}

[1] Pérez-Calpena, A., et al., "MEGARA Fiber bundles”, Proc. SPIE 9147-211, (2014).

[2] Gil de Paz, A., et al., "MEGARA, the new intermediate-resolution optical IFU\&MOS for GTC: getting ready for the telescope", Proc. SPIE 9908-57, (2016). 